\title{
A132 T- AND B CELL CLONES CIRCULATE BETWEEN SYNOVIAL TISSUE AND LYMPH NODE IN EARLY STAGES OF RHEUMATOID ARTHRITIS (RA): ELUCIDATING THE ROLE OF LYMPH NODES IN RA
}

P L Klarenbeek, ${ }^{1}$ M J de Hair, ${ }^{1}$ M E Doorenspleet, ${ }^{1}$ S Alivernini, ${ }^{1}{ }^{2}$ B D C van Schaik, ${ }^{3}$ M G van de Sande, ${ }^{1} \mathrm{~F}$ H Berger, ${ }^{4}$ A H C van Kampen, ${ }^{3}$ D M Gerlag, ${ }^{1} \mathrm{~F}$ Baas, ${ }^{5}$ P P Tak, $\mathrm{N}$ de Vries ${ }^{1}$ Division of Clinical Immunology and Rheumatology, Academic Medical Center, Amsterdam, The Netherlands; ${ }^{2}$ Department, Division of Rheumatology, Catholic University of the Sacred Heart, Rome, Italy; ${ }^{3}$ Department of Clinical Epidemiology, Biostatistics and Bioinformatics, Academic Medical Center, Amsterdam, The Netherlands; ${ }^{4}$ Department of Radiology, Academic Medical Center, Amsterdam, The Netherlands; ${ }^{5}$ Department of Genome Analysis, Academic Medical Center, Amsterdam, The Netherlands

\subsection{6/ard.2010.148999.8}

Background $\mathrm{T}$ - and $\mathrm{B}$ cell receptor (TCR, BCR) mediated responses are likely to play an important role in the initiation of rheumatoid arthritis (RA). The authors recently presented a new high-throughput protocol that can be used to find and quantify T- and B cell expansions. Previous observations from their group suggested that sites other than the synovial tissue (ST) might be involved in the earliest phases of the disease. Therefore, the authors examined the TCR and BCR repertoire in the lymph nodes (LNs) of early RA patients.

Objective To characterise the TCR and BCR repertoire in LN and ST, and investigate overlap in clones between these sites. Methods mRNA was isolated from paired LN, ST and peripheral blood (PB) samples of 2 disease-modifying antirheumatic drug-naive anti-cyclic citrullinated peptide antibody positiveRA-patients after arthroscopic ST sampling of an inflamed ankle joint and biopsy sampling of a regional LN. The disease duration in both patients was $<1$ year. TCRs (for both pts) and BCRs (for $1 \mathrm{pt}$ ) were (linearly) amplified using primers for all $\mathrm{V}$ (ariable genes) of the TCR- $\beta$-chain and BCR-heavy-chain. The samples were processed using a Genome Sequencer (454). The frequency of each clone was determined by its CDR3frequency ( $\%$ of total). Clones $>0.5 \%$ were arbitrarily considered as highly expanded clones (HECs).

Results The LN samples showed $>1000$ B- and T cell clones, but only few HECs were detected (average 8 ( $\mathrm{T}$ cells) and 25 (B cells), respectively). The ST-samples showed strikingly more $\mathrm{T}$ cell HECs (41), but a comparable number of B cell HECs (15). The HECs reached frequencies of up to $20 \%$ (T cells) and $7 \%$ (B cells) in LN compared to $14 \%$ and $36 \%$, respectively, in ST. The HECs were far more dominant in ST where the cumulative frequency of the HECs was $82 \%$ (T cells) and $57 \%$ (B cells) of the analysed cells while this was only $16 \%$ and $35 \%$ in LN. HECs were generally not shared between LN, ST and PB, but there was clear evidence of circulation. $25 \%$ of the ST T cellHECs were also detected in LN (as low frequency clones), while this was $40 \%$ for ST B cell-HECs.

Conclusions HECs ( $\mathrm{T}$ - and B cell) can be detected in both ST and LN of RA-patients. Up to $40 \%$ of the ST HECs are also found as low frequency clones in LN. These results may be explained by circulation of clones between ST and LN. 